formance, a noise and interference test set was used to measure BER (bit error ratio) against $E_{b} / N_{0}$ (bit energy/noise). During these measurements a constant signal level was maintained into the QPSK demodulator $(-37 \mathrm{dBm})$ as its performance was power dependent.

Results: Fig. $2 a$ shows the RF spectrum applied to the laser transmitter (point A, Fig. 1), i.e. TV and $120 \mathrm{Mbit} / \mathrm{s}$ QPSK data. Fig. $2 b$ shows the received QPSK data channel prior to $60 \mathrm{GHz}$ upconversion and radio transmission (point B, Fig. 1)

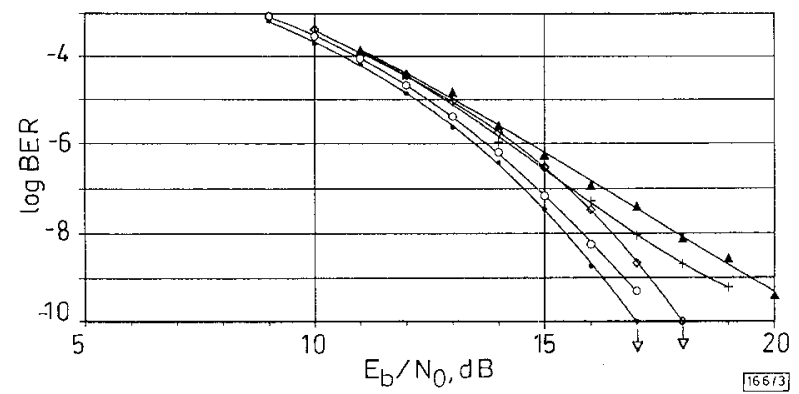

Fig. 3 Bit error ratio against $E_{b} / N_{0}$ at various stages of radio-fibre system

back-to-back

$13 \mathrm{~km}$ CO-RAP link, at output of EAM

A CO-RAP link and $60 \mathrm{GHz}$ RAP-MT link (omnidirect. antenna)

+ CO-RAP link and $60 \mathrm{GHz}$ RAP-MT link (horn antenna)

$12.8 \mathrm{~km}$ RAP-CO uplink

In Fig. 3, we plot the measured BER at different stages of the link. The back-to-back measurement was performed using the output of the first up-converter at $1.35 \mathrm{GHz}$ (point $\mathrm{A}$. Fig. 1) and feeding it directly to the down-converter. There was a $1 \mathrm{~dB}$ penalty at $10^{-7}$ BER on the received QPSK signal at the output of the EAM (point B). Comparing this with the results obtained in [3], this penalty is thought to be due to the narrower bandwidth of the multiplexer in this experiment, resulting in some spectral shaping of the QPSK data. Residual signals from the adjacent TV channels can also be seen in Fig. 2b. For the uplink, (data centred at $140 \mathrm{MHz}$ ) there was a minimal $0.25 \mathrm{~dB}$ power penalty as expected from [3]. The insertion loss of the RAP-MT link added a negligible penalty using the horn antenna, whereas the omnidirectional antenna led to a further $0.7 \mathrm{~dB}$ penalty at $10^{-7}$ BER. The latter penalty is believed to be due to multipath propagation in the laboratory. The presence of reflections was confirmed when the TV channels were transmitted instead of data and these could be received satisfactorily by the MT without using line of sight propagation paths. It is possible that this problem could be alleviated in future experiments by the use of a spread spectrum technique or by using adaptive compensation. BER measurements as low as $10^{-10}$ were observed after $\mathrm{mm}$-wave transmission and a minimum of $10^{-9}$ was achieved in all cases. Additionally, no significant degradation of the TV signals received at the RAP could be observed on the TV monitor:

Conclusion: We have demonstrated bi-directional analogue data transmission at $120 \mathrm{Mbit} / \mathrm{s}$ together with broadcast TV distribution using an EAM as a remote transceiver. The EAM electrical output was used to perform an indoor radio transmission at $60 \mathrm{GHz}$ using two types of antenna at the radio access point. BERs $<10^{-9}$ were observed. The mm-wave system has a novel and attractive strategy for the return path with a reduced amount of equipment at the RAP. Higher bandwidth EAMs could eliminate the need for a separate broadband photodiode by also detecting the $60 \mathrm{GHz}$ carrier, thus enabling the RAP to be further simplified. The configuration we have adopted shows a high degree of functionality and illustrates how a broadband mobile system might be integrated with an optical distribution network.

Acknowledgments: The authors thank P. Sully, J. Reed, M. Roberts, D. Wake and D. Marcenac.

Electronics Letters Online No: 19970857

L. Noël, L.D. Westbrook, D.G. Moodie and D. Nesset (BT Laboratories, Martlesham Heath, Ipswich IP5 $7 R E$, United Kingdom)
References

VOÉL. L., MARCENAC, D., and WAKE, D: 'Optical millimetre-wave generation technique with high efficiency, purity and stability", Electron. Lett., 1996, 32, pp. 1997-1998

2 NOEL. L., MARCENAC. D., and WAKE, D.: " $120 \mathrm{Mbit} / \mathrm{s}$ QPSK radiofibre transmission over $100 \mathrm{~km}$ of standard fibre at $60 \mathrm{GHz}$ using a master/slave injection locked DFB laser source', Electron. Lett., 1996, 32, pp. $1895-1897$

3 WESTBROOK, L.D., NOËL, L., and MOODIE, D.G.: 'Full-duplex $120 \mathrm{Mbit} / \mathrm{s}$ QPSK analogue fibre transmission over $25 \mathrm{~km}$ with simultaneous modulation and detection in an electroabsorption modulator', Electron. Lett., 1997, 33, pp. 694-695

\section{BPSK modulator using VCCS and resonator without carrier signal and balance modulator}

\author{
Juin-Hung Chen and Hen-Wai Tsao
}

Indexing terms: Phase shift keying, Voltage control, Modulators

A new binary-phase-shift-keying (BPSK) modulator is presented. It consists of a voltage control current source (VCCS), an LC resonator and a comparator and is named a switch-resonator BPSK modulator. This type of modulator is simpler than the traditional architectures. The experimental results are also reported; the bit rate and carrier frequency are $2.5 \mathrm{Mbit} / \mathrm{s}$ and $5 \mathrm{MHz}$, respectively.

Introduction: BPSK (binary-phase-shift-keying) modulation is widely used in communication systems. Balanced modulators, implemented by a double balanced Gilbert cell [1] or a diode array and transformer [2], are usually employed to perform BPSK modulation. Two novel methods, using a $180^{\circ}$ phase shifter [3] and a phase splitter circuit [4], were proposed in the microwave band. In this Letter, a novel BPSK modulator, comprising a VCCS (voltage control current source), a resonator and a limiting amplifier, is presented. This modulator does not need a carrier signal, phase shifter or switch circuit. The only input signal is the modulating data bit stream. A short-circuited $\lambda / 4$ line can be used to implement the resonator in the microwave band. This simple architecture is suitable for wireless communication.
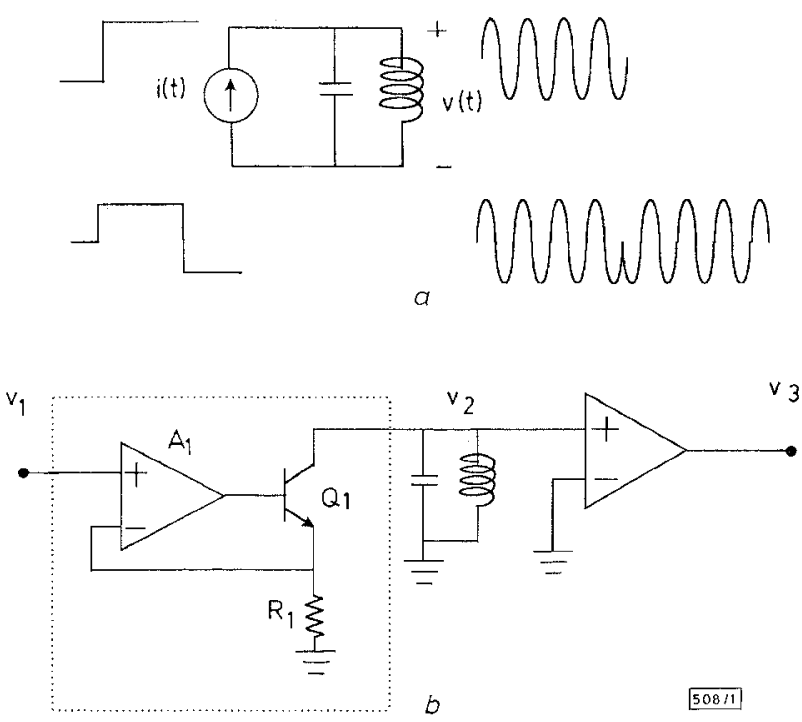

Fig. 1 Proposed configuration and experimental circuit

a Principle of this method

$b$ Experimental circuit

Principle and circuit description: A parallel LC resonant circuit and current source are shown in Fig. 1a. From circuit theory, if the excitation signal is a current step $i(t)=A u(t)$, the voltage across the capacitor (or inductor) will be $v(t)=\left(A / \omega_{0} c\right) \sin \left(\omega_{0} t\right) u(t)$. Using 
this result and the superposition theory, when the excitation signal is $i(t)=A u(t)-A u\left(t-8 \pi / \omega_{o}\right)$, the output voltage will be

$$
v(t)=\frac{A}{\omega_{0} c}\left\{\sin \left(\omega_{0} t\right) u(t)-2 \sin \left[\omega_{0}\left(t-\frac{8 \pi}{\omega_{0}}\right)\right] u\left(t-\frac{8 \pi}{\omega_{0}}\right)\right\}
$$

where $f_{0}=\omega_{0} / 2 \pi$ is the resonant frequency. This signal is also shown in Fig. $1 a$ and we can see that it is identical to a BPSK signal. Therefore, we can employ this concept to design a BPSK modulator.

Fig. $1 b$ depicts the novel BPSK modulator circuit. The VCCS, made by $A_{1}, Q_{1}$ and $R_{1}$, produces the NRZ current pulse stream and injects such a signal into the LC resonant circuit. If the resonant frequency $f_{o}$ is an integral time of the bit rate, the resonator output voltage will be a BPSK signal. Since the quality factor $Q$ of a general purpose reactive inductor is $<100$ and the transistors output resistance is finite, the voltage will decay as time passes. Because BPSK modulation is one kind of exponential modulation, it is immune to system nonlinearity. Therefore, by adding a comparator or limiting amplifier following the resonator, we can compensate for the amplitude decay and still maintain the phase reversal. Passing such a signal through a bandpass filter can produce a filtered BPSK signal as in traditional modulation methods.

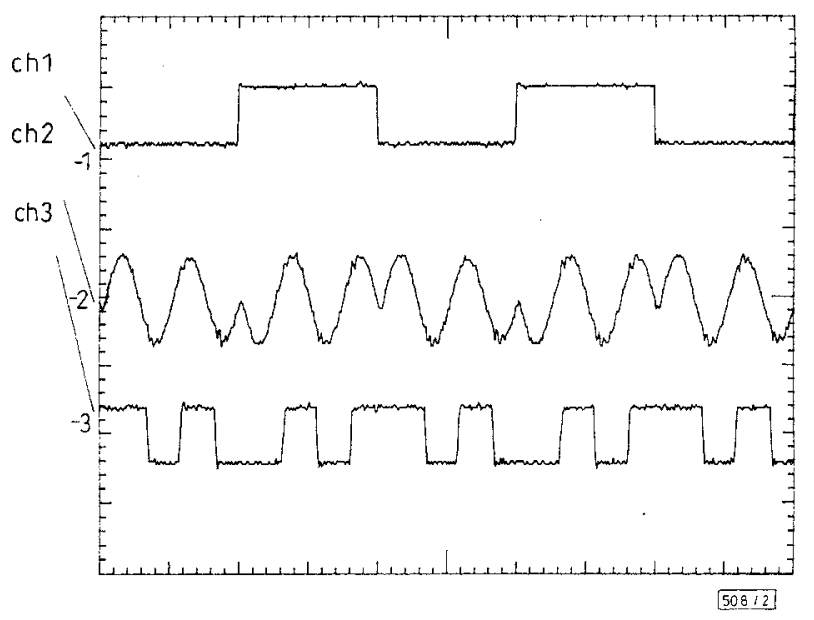

Fig. 2 Resultant experimental waveforms

ch 1: modulating signal $v_{1}$

ch 2: resonator output voltage $v_{2}$

ch 3: comparator output voltage $v_{3}$

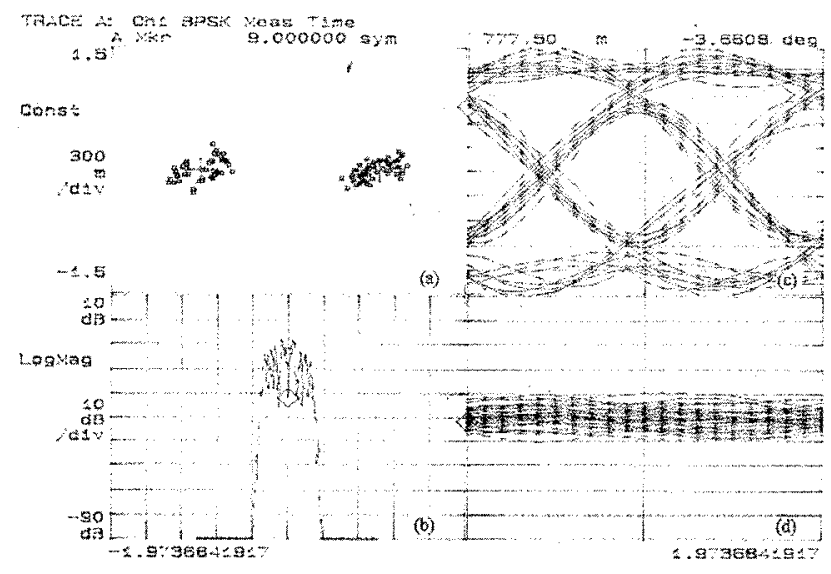

Fig. 3 Spectrum of filtered BPSK signal and demodulation output

a Signal constellation

$b$ Modulator output spectrum following BPF

$c$ Eye diagram of in-phase channel

$d$ Eye diagram of quadrature-channel

Experiment result: Channel 1 in Fig. 2 is the modulating signal $v_{1}$ in Fig. $1 b$; channel 2 is the resonator output voltage $v_{2}$; channel 3 is the comparator output voltage $v_{3}$. We find that phase reversals occur at the transition of the modulation data bits. To appraise this modulator performance, we use a PN signal (with length $2^{10}-1$ ) generated from a digital transmission analyser (Anritsu ME520A) as the modulating signal $v_{1}$, and use a vector signal analyser (HP89441) to demodulate the BPSK signal from the switch-resonator BPSK modulator. Fig. $3 a$ shows the signal constellation;
Fig. $3 b$ shows the spectrum of the filtered BPSK signal (centre frequency $5 \mathrm{MHz}$; bandwidth $2.5 \mathrm{MHz}$ ). Fig. $3 c$ is the eye diagram in the in-phase channel and Fig. $3 d$ is the eye diagram in the quadrature-channel. This Figure shows that the signal generated from the switch-resonator BPSK modulator is correct.

Conclusion: A novel BPSK modulator has been implemented by using a current source, an $\mathrm{LC}$ resonator and a comparator without an additional carrier signal, balanced modulator or phase shift circuit. This modulator is easy to assemble and its performance is acceptable. Except for the resonator, the VCCS and the limiting amplifier (or comparator) can be packaged in an IC and a high bit rate BPSK signal can be directly generated. It is suitable for generating direct sequence spread spectrum (DSSS) signals in wireless communication.

\section{(C) IEE 1997}

Electronics Letters Online No: 19970885

2 June 1997

Juin-Hung Chen and Hen-Wai Tsao (Room 213, Department of Electrical Engineering, National Taiwan University, Taipei, Taiwan 107, Republic of China)

\section{References}

1 Harris Corporation: 'PC card wireless LAN handbook'. 1996, pp. 61.62

2 YOUNG, P.H.: 'Electronics communication techniques' (Macmillan, New york, 1994)

3 MAZUMDER, S.R., and WATERMAN, R.C.: 'A novel 6 to $18 \mathrm{GHz} 180^{\circ}$ bit phase shifter configuration having very small amplitude and phase errors'. IEEE MIT-S Int. Microw. Symp. Dig., 1994, Vol. 1, pp. 83-86

4 GOLDFARB, M.E, BRADFORD COLE, J., and PLATZKER, A.: 'A novel MMIC biphase modulator with variable gain using enhancementmode FETS suitable for $3 \mathrm{~V}$ wireless application'. IEEE Microw. and Millimetre-Wave Monolithic Circuit Symp., 1994, pp. 99-102

\section{Correlation model for shadow fading in land- mobile satellite systems}

\author{
P. Taaghol and R. Tafazolli
}

\begin{abstract}
Indexing terms: Satellite communication, Correlation methods
A simple yet accurate correlation model for shadow fading in land-mobile satellite systems, derived from L and S-band channel recordings, is proposed. The model has been developed for heavily wooded and suburban environments and covers elevation angles of $60-80^{\circ}$. It is demonstrated that in such environments, the effective correlation distance of shadowing is in the order of a few tens of meters.
\end{abstract}

Introduction: Recently, there has been a great effort in the characterisation of the land-mobile satellite propagation channels, enabling the system design and dimensioning of the proposed satellite personal communication networks (S-PCN). Such experiments are very costly and time consuming. Consequently, unlike the terrestrial case, a very limited number of such campaigns have been carried out. The first-order (time-invariant) statistics of the landmobile satellite propagation channel based on experimental results have been extensively reported in [1] and [2], however, the secondorder (time-variant) statistics are generally much less well-known; correlation statistics of shadow fading are one example, and have not yet been made available for satellite channels, restricting research [3] to only terrestrial values reported by [4]. An accurate correlation model for shadow fading in such environments is of vital importance for both analytical and simulation based evaluation of any power control and handover scheme.

Channel recordings: The proposed shadow fading correlation model is based on the narrowband measurement campaign recordings at L-band $(1.3 \mathrm{GHz})$ and S-band $(2.3 \mathrm{GHz})$, carried out by CCSR of University of Surrey in spring 1992 [1]. The measure- 